\title{
EMOCIONES, VALORES Y MORAL
}

\author{
Holmer Steinfath $^{*}$ \\ doi:10.11144/Javeriana.uph31-63.evms
}

\begin{abstract}
RESUMEN
En los debates metaéticos actuales, numerosos autores creen que existe una estrecha relación entre las emociones y los valores. Algunos piensan que las emociones ayudan en la constitución de los valores morales y no morales. Otros ven las emociones como instrumentos epistémicos para adquirir conocimiento sobre los valores. Ambas perspectivas tienen raíces que se adentran bien atrás en la historia de la filosofía, y las dos pueden ser desarrolladas tanto para apoyar como para socavar las teorías realistas y anti-realistas de los valores. Este artículo pretende esclarecer una complicada situación dialéctica. Adicionalmente, se realiza una propuesta constructiva encaminada hacia una mejor comprensión de la conexión entre emociones y valores. La propuesta, aunque es anti-realista en espíritu, ha sido diseñada para integrar también algunas importantes intuiciones realistas.
\end{abstract}

Palabras clave: emociones; valores; realismo valorativo; deseos; juicios de valor

\footnotetext{
${ }^{*}$ Georg-August-Universität, Gotinga, Alemania.

RECIBIDO: 01.04.14

ACEPTADO: 16.05 .14

DISPONIBLE EN LÍNEA: 02.12 .14

La presente traducción al castellano, realizada por Johnny Antonio Dávila, Universidad Antonio Nariño (Bogotá-Colombia) y autorizada por el autor para su publicación, procede de: Steinfath, H. (2002). Emotionen, Werte und Moral. S. Döring. \& V. Mayer. (Hrgs.), Die Moralität der Gefühle (pp. 105-122). Berlin: Akademie-Verlag.

Para citar este artículo: Steinfath, H. (2014). Emociones, valores y moral. Universitas Philosophica, 31(63), pp. 71-96, ISSN 0120-5323, ISSN en línea 2346-2426, doi: 10.11144/ Javeriana.uph31-63.evms
} 
UNIVERSITAS PHILOSOPHICA, 31(63), pp. 71-96

julio-diciembre 2014, Bogotá, Colombia - ISSN 0120-5323

\title{
EMOTIONS, VALUES AND MORAL
}

\author{
Holmer Steinfath
}

\begin{abstract}
In current meta-ethical debates, many authors believe that there is a close relation between emotions and values. Some think emotions help to constitute moral and non-moral values. Others see emotions as epistemic devices to gain knowledge of values. Both views have roots reaching well back into the history of philosophy, and both can be developed to either support or undermine realistic and anti-realistic theories of value. The article aims to clarify a complicated dialectical situation. In addition, it makes a constructive proposal towards a better understanding of the connection between emotions and values. Though anti-realistic in spirit, this proposal is designed to integrate some important realistic intuitions as well.
\end{abstract}

Key words: emotions; values; value-realism; desires; value judgment 


\section{Introducción}

A CONTINUACIÓN QUISIERA EXAMINAR ALGUNAS CONEXIONES - esencialmente conceptuales- entre las emociones, los valores y la moral. Mis reflexiones se dividen en seis partes: en la primera sección aclararé qué entiendo por emociones y qué entiendo por valores. En la segunda, examino muy concisamente diferentes teorías relativas a la determinación de las relaciones entre valores y emociones. Según la teoría a la que le otorgo preferencia, algo es valioso cuando puede ser objeto de una emoción idónea. Esta visión da lugar a la pregunta cuándo y en qué sentido puede decirse que las emociones son idóneas. En la tercera y en la cuarta parte, proporciono elementos para dar una respuesta a esta pregunta; estos constituyen el centro de mis observaciones. En la quinta parte, presento reflexiones sobre la posibilidad de aplicar a la clase especial que constituyen los valores morales la teoría de los valores desarrollada hasta ese momento. En la sexta y última parte, concluyo mi exposición con una observación sobre la relación entre los valores morales y las normas morales, las cuales deben ser distinguidas de dichos valores ${ }^{1}$.

\section{Emociones y valores}

TANTO EN LA FILOSOFÍA COMO EN LAS RAMAS DE LA PSICOLOGÍA está extendida la opinión de que existen importantes relaciones entre las emociones y los valores $^{2}$. No obstante, los acuerdos de quienes conceden a las emociones un papel principal para la explicación de los valores son, frecuentemente, de naturaleza superficial. Ya en la diferenciación de las emociones con respecto a otros estados y actitudes (1) y en la caracterización de los valores (2) se comienza con disputas.

\footnotetext{
${ }^{1}$ Ya en otras dos publicaciones me he ocupado de la relación entre emociones -dicho más ampliamente, sentimientos- y valores. Véase: Steinfath (2001a, cap. 4 y 2001b, pp. 196-220). Las convergencias y divergencias con esas dos publicaciones solo se señalarán esporádicamente en este escrito. Tanto las reflexiones previas como las que se presentan aquí las considero exploraciones tentativas en la vía hacia una teoría más amplia, que espero poder presentar en algún momento.

${ }^{2}$ Entre tanto, la literatura relevante sobre el tema apenas puede ser abarcada con la vista. En representación de estos trabajos filosóficos pueden ser nombrados: E. Anderson (1993), R. de Sousa (1987) y M. Nussbaum (1996).
} 
(1) Fenómenos como miedo, molestia, vergüenza, repugnancia, alegría, duelo, indignación, admiración y envidia, los considero emociones típicas. $\mathrm{Si}$ uno los toma como estados o actitudes actuales entonces hay, por lo menos, dos propiedades que le son características. Primero, cada una de ellas está conectada, aunque no necesariamente, con su propia y distinta forma de sentir, percibir o de ser afectado. Dicho en la jerga de la filosofía del espíritu: las emociones son qualia o están esencialmente unidas con qualia. Yo quisiera denominar afectividad al lado sensible de las emociones. Segundo, las emociones tienen implicaciones cognitivas que están más o menos claramente delineadas; ellas poseen un contenido representacional. Afectividad es aquella propiedad de las emociones que las distingue de actitudes como opiniones o intenciones, mientras que el contenido cognitivo permite diferenciarlas de otros fenómenos afectivos como instintos, sensación puramente corporal y disposiciones individuales.

Que la afectividad es un rasgo elemental de las emociones, se corresponde con una extendida comprensión que se tiene de ellas en la vida cotidiana, comprensión que, sin embargo, con mucha frecuencia se refuta en la filosofía ${ }^{3}$. Así, se ha llamado la atención sobre el que alguien pueda llorar durante años la pérdida de un amigo, sin encontrarse por ello en un estado de permanente depresión (Oakley, 1992, p. 8). Los seres humanos pueden también estar celosos, sin ser conscientes de ello. ¿Y no hay formas del amor que, a diferencia del enamoramiento, se manifiestan totalmente sin sensaciones perceptibles? Pero contraejemplos de este tipo no son muy convincentes. Algunas veces atribuimos a las personas emociones, aunque sería más preciso hablar de un estar inclinado hacia emociones. Una persona irascible, por ejemplo, se inclina a molestarse frecuentemente. A diferencia de un intenso arrebato de cólera, él no tiene por qué percibir su disposición hacia tales arrebatos. Pero si uno desea aclarar qué significa estar inclinado hacia una emoción, se debe saber qué significa poseer intensamente tal emoción ${ }^{4}$. Por esta razón, yo quisiera reservar la expresión emociones para sensaciones presentes, y no en el sentido de estados y actitudes hacia los que uno se inclina. Pero con este modelo no pueden ser desestimados todos los contraejemplos. En este sentido, el duelo largo y permanente es más que la

\footnotetext{
${ }_{3}^{3}$ Por ejemplo, véanse: Bedford (1957, pp. 281-304/283), Pitcher (1965, pp. 326-346/338), Lyons (1980, p. 58) y Solomon (1993, pp. 96-102).

${ }^{4}$ Lyons argumenta de modo similar (1980, pp. 53-57).
} 
disposición hacia asaltos de tristeza, sean ocasionales o frecuentes. Mas en este caso hay un matiz subjetivo de larga duración de la vida por parte de la persona que guarda duelo, el cual ella muy bien puede percibirlo, aunque tal vez solo muy atenuadamente. También en el caso de las emociones que duran poco tiempo sucede que las sensaciones que le son correspondientes yacen más bien en los márgenes del campo de nuestra conciencia. A manera de ejemplo, podemos experimentar tanta dicha en una actividad, que apenas captamos nuestra dicha. De igual manera, una situación aterradora puede ejercer tal influencia sobre nosotros, que apenas sentimos nuestro miedo o terror. A pesar de ello, estos estados se diferencian fenoménicamente de aquellos sin dicha o miedo. Estos muestran los cambios que percibimos, tan pronto la dicha disminuye o el miedo se disipa. Inclusive estados emocionales relativamente distanciados, como el estar interesado en algo, tienen un componente perceptivo, aunque casi siempre muy débil. Por el contrario, un caso límite lo representan las actitudes más estables de una persona, que pueden ser especificadas con expresiones de emoción, como el amor hacia una persona o también hacia un objeto o una actividad. En ellas la faceta sensorial juega a veces un papel muy supeditado. También aquí solo resta preguntar si el aspecto sensitivo puede permanecer al margen. El amor sin ninguna sensación de cariño, de simpatía, de agrado, etc. difícilmente se merece ese nombre.

Si la afectividad de las emociones puede existir de diferentes maneras y con diferente intensidad, entonces también sus implicaciones cognitivas son de diferente tipo y complejidad. A menudo las emociones están fundadas en juicios de valor; en ellas se puede expresar de hecho cómo una persona valora el mundo en su totalidad. De igual manera, con frecuencia las opiniones descriptivas son elementos o condiciones para la aparición de una emoción. Así, la indignación moral hacia otra persona normalmente asume la presunción de que esa persona consciente o voluntariamente ha hecho o dejado de hacer algo determinado, así como el juicio de que esta ha violado con ello una norma importante. Sin embargo, a diferencia de quienes sostienen las teorías emotivistas impregnadas de cognitivismo, no creo que el contenido cognitivo de las emociones siempre esté estructurado proposicionalmente y ni que esté unido con una pretensión de verdad. A veces es suficiente con una simple percepción sensorial -la cual tengo por un estado cognitivo no proposicional y no cognitivo- para desencadenar una emoción (Peacocke, 2001, pp. 239-264). De este modo, el solo ver una culebra puede provocar miedo, mas la creencia de que las culebras son 
peligrosas se forma, dado el caso, solo posteriormente, tal vez únicamente en razón del miedo. El encuentro con un bello paisaje o con una persona atractiva puede suscitar agrado o admiración, aunque el juicio según el cual paisajes y personas son bellos y atractivas se emite únicamente después, y no en pocas ocasiones solamente debido a la correspondiente emoción.

Afectividad y contenido cognitivo no son las únicas características de las emociones: la mayoría de ellas tienen un lado motivacional. Frecuentemente las emociones van acompañadas por expresiones faciales, y probablemente también tengan una base neuronal distintiva. No obstante, para mi propósito es suficiente con definir las emociones como estructuras afectivo-cognitivas.

(2) ¿Qué se debe entender por valores? Hasta hoy día la expresión valor no tiene un lugar fijo en el habla coloquial, de forma que aquí se debe definir un poco más que en el caso de las emociones. Parto del supuesto de que algo o es un valor o tiene un valor, cuando ello puede ser caracterizado con la ayuda de uno o varios predicados valorativos. En esto se pueden distinguir dos tipos de predicados valorativos. Primero, existen los predicados generales y no específicos bueno y malo, así como sus comparativos y superlativos. Segundo, hay predicados más específicos o compactos como feo, sublime, repugnante, valiente, admirable, amigable, generoso y elegante. De qué manera ambos tipos de predicados están relacionados entre sí, es algo que dejo abierto ${ }^{5}$. Los mencionados ejemplos de predicados valorativos específicos y no específicos dejan claro que quiero asociar el discurso sobre los valores tanto con valores positivos como con negativos.

La adscripción de valores tiene lugar pues, cuando alguien, desde alguna perspectiva, estima algo altamente o poco, lo tiene por bueno o lo juzga como malo. Me parece sensato partir primero de un concepto bastante amplio de estimación y valoración, el cual se extiende desde sencillas expresiones de agrado y desagrado hasta juicios elaborados. De acuerdo con esto, hay innumerables cosas que son candidatas para ser valores u objetos de valor. Los seres humanos estiman la naturaleza virgen, las obras de arte, la amistad y el amor, la autonomía y la justicia, la paz y la vida. Aunque también estiman cosas más banales como autos, series de televisión y el

\footnotetext{
${ }^{5}$ En Millgram (1995, pp. 354-388) pueden encontrarse: una propuesta sobre este punto, valiosos conceptos éticos y la diferenciación entre valores y hechos.
} 
chocolate, y algunas estiman la destrucción, la violencia y la guerra. A veces se habla de los valores de una persona. Se trata, entonces, generalmente de las cosas que más cuentan para la persona en cuestión, cosas por las que se preocupa y por las que se esfuerza especialmente.

Los valores y las expresiones valorativas se encuentran en un claro contraste con las normas y las expresiones normativas como deber y tener que. Kevin Mulligan (1998, pp. 161-188), en su instructivo ensayo From Appropriate Emotions to Values, ha llamado la atención sobre tres importantes diferencias entre expresiones valorativas y normativas. De esta manera, la clase de las expresiones valorativas contiene predicados relacionales como "_mejor que_" y "_peor que_", mientras que no existe un equivalente en el habla cotidiana para las expresiones normativas. En especial, no hay un comparativo para la expresión deber actuar de esta y esta manera (Mulligan, 1998, p. 164). Uno, o debe hacer algo, o no debe hacerlo, o está permitido. Pero nada está más o menos prohibido, a pesar de que puede ser más o menos malo -o llegar a ser castigado con sanciones más o menos fuertes- realizar algo prohibido. Consecuencialmente, en el campo de las expresiones normativas no pareciera haber nada paralelo a la diferenciación entre predicados valorativos específicos y no específicos. Y, finalmente, al menos los predicados valorativos específicos o compactos tienen un contenido descriptivo, con respecto a lo cual igualmente no existe nada comparable en las expresiones normativas. Como Mulligan destaca (1998, p. 165), es bastante evidente suponer que la posesión de una propiedad específica -o compacta- axiológica implica la posesión de una determinada propiedad natural. Por ejemplo, al ser valiente le corresponde, entre otras cosas, conocer el miedo y superarlo o no dejar que surta efecto. Por el contrario, los enunciados sobre lo que se debe hacer y lo que se debe dejar no contienen suposiciones del mismo tipo sobre propiedades naturales.

Estas alusiones ayudan a mantener separado lo valorativo de lo normativo. No confundir ambas cosas es importante, ya que frecuentemente justificamos normas con el recurso a los valores. Por ejemplo, podemos opinar que uno no debería matar, pues la vida es valiosa. Rige, así, que cuando estamos confrontados con diferentes alternativas para actuar debemos escoger aquella que es la mejor. 


\section{Los valores como objeto de emociones idóneas}

LUEGO DE ESTAS CLARIFICACIONES PRELIMINARES, puedo centrarme ahora en las relaciones entre emociones y valores. ¿Por qué puede, en realidad, ser obvio aceptar que existe una estrecha relación entre ambos?

El argumento más contundente es de naturaleza lingüística. En todas las lenguas que conozco existe un vocabulario increíblemente rico para la adscripción de valores positivos y negativos, que semánticamente remite a emociones. Esto ciertamente no concierne a las expresiones valorativas generales y no específicas bueno y malo. Aunque ello puede ser constatado en muchos predicados valorativos específicos o compactos. Algunos de ellos ya los he mencionado, como por ejemplo: grato, aburrido, repugnante, admirable, encantador, indignante. Por lo menos en el caso de estas -de ninguna manera marginales- expresiones valorativas salta a la vista la idea de que las emociones juegan un papel importante en relación con los valores. Una suposición de este tipo también parece plausible de cara a las expresiones valorativas que ciertamente no apuntan directamente a emociones determinadas, pero a las cuales se les puede adjudicar emociones. Un ejemplo de ello son los predicados valorativos estéticos como feo y sublime, pues lo feo desagrada de manera particular, mientras que lo sublime puede provocar admiración y pavor. De igual manera pueden ser contemplados injusticia y crueldad como objetos naturales de la indignación; grandes obras de arte y seres humanos con capacidades especiales, en tanto objetos naturales de admiración, y lugares religiosos, en tanto objetos de veneración.

Ejemplos de este tipo sirven de alimento a la concepción de que para nosotros el mundo sería un lugar que posee mucho menos valor -si es que inclusive ningún valor-, si a todos nos fueran robadas nuestras emociones. Sin emociones, así parece, todo nos sería indiferente; por decirlo de alguna manera, todo se sumergiría en una luz fría, neutral. Pienso que esta intuición es correcta, y que seres sin emociones perciben el mundo, cuando menos, como mucho menos valioso que los seres que disponen de emociones. Esto es básicamente compatible con un conjunto de diferentes explicaciones teóricas sobre la conexión entre emociones y valores.

Sin poder fundamentarlo aquí más detalladamente, yo quisiera refutar dos planteamientos teóricos que están en marcado contraste uno con otro y que han sido desarrollados en la primera mitad del siglo XX. El 
primero de estos planteamientos es el de un realismo estricto o ingenuo. Según esta concepción, los objetos tienen propiedades axiológicas que son independientes del espíritu y del observador, las cuales podemos captar con ayuda de determinadas emociones. De acuerdo con ello, las emociones son entendidas como actitudes que nos procuran un acceso epistémico hacia los valores en tanto partes especiales de la realidad objetiva. Aquí la correspondencia entre emociones y valores se modela conforme con el patrón de la relación entre percepción óptica y colores. Un punto de vista tal es sostenido, por ejemplo, por Scheler ${ }^{6}$. Con vista en Moore, J. L. Mackie (1977, pp. 138 ss.) ha criticado convincentemente una versión de este punto vista en su libro Ethics. A pesar de todas las debilidades de la argumentación de Mackie, es justificado el reproche de que un estricto realismo valorativo, el cual trata los valores como cualidades primarias o también secundarias ${ }^{7}$ de los objetos, no tiene ningún puesto en una ontología compatible con la ciencia moderna.

La segunda teoría -absolutamente opuesta- es el emotivismo radical del joven Alfred Ayer (1936, cap. 6). Si uno sigue esta teoría, las valoraciones no son otra cosa que expresión de estados emocionales subjetivos o actitudes, y los valores son solamente proyecciones en el mundo de estos estados o actitudes. Mientras que el realismo opera con una ontología singular(Mackie), el emotivismo descuida importantes rasgos de muchas valoraciones, como su impugnabilidad y su pretensión de objetividad. No solo en el caso de juicios de valor morales, sino también en el de muchos juicios de valor estéticos suponemos la posibilidad de poder justificar nuestros juicios de una manera como no lo hacemos en las cuestiones del gusto.

Un tercer enfoque, sostenido en forma de diferentes concepciones valorativas disposicionales ${ }^{8}$, está afectado por problemas muy similares. Según este planteamiento, algo tiene un valor cuando tiende a producir

\footnotetext{
${ }^{6}$ Sobre este punto, véase: Scheler (1980). Algunas reflexiones de De Sousa (1987) y Johnston (2001, pp. 181-214) siguen una orientación como la de Scheler.

${ }^{7}$ Sobre la comparación entre cualidades secundarias como colores y valores, consúltese McDowell (1998, pp. 131-150). Una posición crítica se encuentra en Steinfath (2001b, pp. 202-204).

${ }^{8}$ Lewis (1989, pp. 113-137) sostiene una versión de las teorías valorativas disposicionales, la cual, sin embargo, no se refiere a las emociones.
} 
una determinada reacción emocional en el observador. También para ello el modelo de la percepción del color puede ser un referente. Así, en analogía con una difundida interpretación de la adscripción del color, uno podría opinar que algo es admirable o indignante cuando personas medias, bajo condiciones normales, tratan ese algo con indignación o admiración. Podría, pues, ser posible solucionar disputas relativas a valores sobre la base de investigaciones puramente empíricas. Esta propuesta tiene una cierta plausibilidad en lo que respecta a predicados valorativos emocionalmente matizados, comolo son asqueroso y aburrido. Aunque es incorrecta en relación con predicados como admirable, indignante y aborrecible. Adscripciones de este tipo pueden ser sostenidas con argumentos; nosotros comprobamos regularmente su exactitud y las empleamos para guiar nuestros propios sentimientos y actos y los de los demás. Estas adscripciones están abiertas a justificaciones de una forma que puede ser captada sin ninguna referencia a una sólida disposición nuestra o de una mayoría de personas9. Finalmente, también las disposiciones de una mayoría pueden ser rectificadas a la luz de nuevas experiencias, pues ellas pueden estar totalmente equivocadas. Las teorías valorativas disposicionales no poseen una aclaración convincente para la práctica del continuo refinamiento recíproco de nuestras reacciones emocionales y de nuestro lenguaje valorativo ${ }^{10}$.

Con ello se pone de manifiesto un doble reto: al contrario del emotivismo y de las teorías disposicionales, debemos tomar en consideración de manera apropiada los aspectos racionales de las valoraciones, pero debemos hacer esto sin recaer en una variante del realismo valorativo ingenuo. Mientras que el emotivismo y las teorías valorativas disposicionales podrían ser correctas, en tanto que por lo menos muchos valores parecen carecer de una base si no disponen de emociones, el realismo luce atractivo, ya que él tiene para ofrecer una explicación de las condiciones de corrección que regularmente se dan por supuestas en los discursos valorativos. La pregunta es cómo pueden ser conjugadas las fortalezas de las tres posiciones, de tal manera que se eludan sus debilidades.

Diferentes autores anhelan precisamente poder dar una respuesta con aquella concepción de valor a la que al comienzo le otorgué preferencia.

\footnotetext{
${ }^{9}$ En este mismo sentido, véase D’Arms y Jacobson, 2000, pp. 722-748/726.

${ }^{10}$ De igual forma piensa Mulligan (1998, p. 178).
} 
Según ella, algo es valioso cuando puede ser objeto de una emoción idónea ${ }^{11}$. Quien opina que un objeto tiene una cierta propiedad valiosa, por lo menos en muchos casos considera con ello que es apropiado adoptar determinada actitud emocional frente al objeto correspondiente ${ }^{12}$. ¿Pero de qué manera las emociones pueden y deben ser idóneas para conducirnos hacia los valores? ¿Y pueden ellas realmente ser de tal forma que se eviten los problemas del emotivismo, del disposicionalismo y del realismo?

\section{La idoneidad de las emociones}

NO PUEDO OFRECER UNA RESPUESTA ACABADA a estas preguntas pero quisiera, por lo menos, marcar la dirección en la cual dicha respuesta, según mi opinión, debe buscarse. La dificultad más grande consiste en la enunciación de criterios para la idoneidad de las emociones, sin que esos criterios hagan superfluas a las emociones mismas, a los fines de la comprensión de los valores. En el examen de idoneidad de las emociones se halla en sí una valoración, y esta pareciera justamente ser independiente de experiencias emocionales. Si se mide el valor de algo en relación con nuestro juicio sobre la idoneidad de una emoción, entonces pareciera que solo nuestro juicio decide sobre este valor, y no la emoción para la cual el juicio es válido.

Un primer paso para la clarificación del discurso sobre la idoneidad de las emociones se ha realizado rápidamente: las emociones son idóneas cuando están justificadas y, por otra parte, la posibilidad de justificarlas se basa sobre la mencionada circunstancia de que las emociones poseen implicaciones cognitivas, más o menos claramente definidas, que les proveen un contenido representacional. Por lo tanto, es apropiado justificar emociones en relación con su contenido cognitivo.

Como primer ejemplo, tomemos el miedo. Como ya lo destaqué, pienso que el miedo en tanto base cognitiva única puede tener una percepción sencilla, en la cual se trata de un enfoque cognitivo no proposicional. Con simplemente ver una cobra que repta alrededor de mis pies siento miedo. Claro que también puedo asustarme porque pienso que una cobra repta

\footnotetext{
${ }^{11}$ D'Arms y Jacobson incluyen a autores como Allan Gibbard, John McDowell y David Wiggins entre los partidarios de esta posición.

${ }^{12}$ Esta formulación la he tomado de D'Arms y Jacobson (2000, p. 729).
} 
alrededor de mis pies. En ambos casos mi miedo se muestra justificado, si la correspondiente percepción o creencia es correcta o verdadera. Si ello es correcto, entonces mi miedo es idóneo con mi situación, lo que significa que mi situación es peligrosa y, en este sentido, es valorada negativamente.

Como segundo ejemplo, quisiera retomar la admiración. Quizá pueda ocurrir que la sola percepción de un objeto -por ejemplo, de una construcción o de una persona- produzca admiración. Adam Smith (1984, p. 20) caracterizó la admiración como una forma de aprobación (approbation), que es realzada por medio del asombro (wonder) y la sorpresa (surprise), y para ello puede ser suficiente una simple percepción. No obstante, mucho más probables son los casos en los cuales nuestra admiración radica en complejas convicciones sobre el objeto que admiramos. En este sentido, podemos admirar las pirámides egipcias porque creemos que fueron creadas sin la técnica moderna y porque hay pocas construcciones de la misma época que se les comparan. De cualquier manera, también la admiración se muestra pues, justificada, si la correspondiente percepción o creencia es correcta o verdadera. Así, se trata de una admiración idónea, y ello significa que el objeto al cual ella se dirige es realmente digno de admiración y que no solamente es tenido por ello.

No pienso que uno deba darse por satisfecho con este análisis que fija la idoneidad de las emociones sencillamente en el contenido de la realidad o en la verdad de los enfoques cognitivos que se incluyen en ellas. A pesar de ello, vale la pena detenerse por un momento en los ejemplos -ciertamente sencillos- del miedo y la admiración, y destacar dos puntos.

El primer punto se refiere al paralelismo entre ambos ejemplos: cada ejemplo lo he formado de tal manera que las mencionadas emociones surten efecto sin que se cimenten necesariamente en convicciones axiológicas o en juicios de valor. Con otras palabras: no debemos creer que algo es peligroso para sentir miedo, y tampoco debemos creer que algo es admirable para admirarlo. Con ello no quisiera refutar que nuestras reacciones emocionales muy frecuentemente están condicionadas por juicios de valor de uno u otro tipo. Mas, para el proyecto de un engranaje - tan íntimo como sea posible-de las emociones y los valores, una dependencia sin excepción o inherente de las reacciones emocionales para con los juicios de valor sería fuertemente perjudicial. Esa dependencia daría sustento al pensamiento de que los valores siempre son algo superior a las emociones y, de esta manera, o bien 
apoyaría nuevamente una forma de realismo valorativo, o bien compelería a desarrollar una concepción de los valores mediante el recurso a otras actitudes de tipo emocional per se. Lo último podría resultar, por ejemplo, de actitudes que fijan metas, como el querer o la intención. Entonces, algo tendría un valor si sirviera a las metas que nos fijamos por medio de actos volitivos (Steinfath, 2001b). Por lo menos un valor, el de lo útil, en gran parte puede ser analizado exactamente así.

El segundo punto que deseo resaltar alude a la significativa diferencia entre la emoción del miedo y la de la admiración. El miedo funge como indicador de peligro. Dado el supuesto de que su base cognitiva se corresponde con la realidad, ella nos ayuda a estar alertas ante el peligro de nuestra situación. Para criaturas como nosotros, la capacidad de temer es esencial para sobrevivir, y en vista de los desarrollos técnicos como la energía atómica y la ingeniería genética, uno puede desear que los seres humanos sean más temerosos de lo que lo son en realidad ${ }^{13}$. Por el contrario, no podemos decir que el miedo constituya un peligro. Algo no se hace peligroso porque nosotros temamos a eso. Fácilmente puede uno pensar en un mundo lleno de peligros en el cual nadie es capaz de sentir miedo. Si el peligro, entendido como un valor negativo, a fin de cuentas está esencialmente unido con una emoción, entonces debe tratarse en ese caso de alguna otra emoción distinta a la del miedo. Por ejemplo, uno podría sopesar si la peligrosidad de una situación para una criatura no supone que para esa criatura algo es emocionalmente valioso, que ella ama algo o que de algún otro modo aprecia eso afectivamente. Pero ello tendría como consecuencia que podríamos hablar únicamente de forma derivativa sobre peligros para criaturas u objetos que no son capaces de sentir.

Estas relaciones se manifiestan de una forma diferente en el caso de emociones como la admiración. Aquí es más difícil imaginarse un mundo con propiedades admirables, sin presumir la existencia de criaturas que puedan admirar algo. Algo similar ocurre con otras emociones como alegría, repugnancia o amor, y con las expresiones de valor que le son

\footnotetext{
${ }^{13}$ En efecto, puede ser que los seres humanos sean concebidos en general de forma que puedan temer únicamente a las amenazas de daño inmediato. Por ello, Aristóteles (Ret. II 5, 1382a35) define el temor como "una especie de dolor o inquietud, en razón de la representación de un mal pernicioso o doloroso que amenaza directamente".
} 
correspondientes, así como con todas las emociones que son relevantes para los juicios estéticos, entendidos estos tanto en sentido amplio como restringido. Esto no excluye necesariamente la posibilidad de que algo sea digno de admiración, a pesar de que nadie lo admire, o de que sea digno de amor, sin que nadie lo ame. Pero parece que, a efectos de la aclaración de eso que es admirable, no tenemos otra opción que apelar al sentimiento de admiración. Lo que es digno de admiración no podemos explicarlo sin referencia a la admiración, así como sí podemos hacer comprensible lo peligroso sin referencia al miedo. En este respecto, lo que es digno de admiración de alguna forma $u$ otra parece estar constituido también por la admiración.

Podemos, por lo tanto, distinguir tentativamente dos funciones diferentes que poseen las emociones en conexión con los valores: algunas emociones sirven como indicadores o indicios de valores; ellas señalan su existencia. Por el contrario, otras juegan un papel constitutivo para los valores. En este caso, la existencia de los valores depende de la existencia de criaturas que tengan ciertas emociones o, por lo menos, la disposición hacia ellas. Esta diferencia se desvanece en concepciones que explican los valores como correlatos de emociones idóneas. Sin embargo, a esta diferencia se le debe dar gran importancia, ya que deja claro que la afirmación de una estrecha relación -en el sentido de algo constitutivo- entre emociones y valores en realidad solo puede ser aplicable a un cierto grupo de emociones y valores.

Todo habla en favor de que esta diferencia esencial entre una emoción como el miedo y una emoción como la admiración tiene repercusiones sobre el problema de la idoneidad de las emociones. La observación de que las emociones pueden ser justificadas en conexión con su contenido cognitivo puede, en este sentido, no más que ser vista como un primer paso en dirección hacia una solución satisfactoria de este problema. La observación se mantiene en la superficie, pues incluso aunque nos mantengamos en nuestros dos simples ejemplos, todavía se puede preguntar por qué la percepción de una cobra o una cierta creencia sobre la construcción de las pirámides en Egipto debe poder justificar la actitud de miedo o admiración.

En cuanto al miedo, se puede dar una respuesta relativamente sencilla. El miedo tiene la función de llamar nuestra atención sobre posibles peligros para nosotros o para personas o cosas que significan algo para nosotros. En esta tarea el miedo fácticamente sólo puede ser sustituido parcialmente por 
medio de formas de valoraciones situacionales que son no emocionales. Por lo tanto, el miedo tiene un indiscutible uso instrumental. Al mismo tiempo, este uso pone a disposición un claro parámetro para la valoración de su idoneidad. El miedo cumple con su función de hacer consciente al sujeto asustado de los peligros para cosas que le son importantes y, si es posible, de tal forma que pueda tomar medidas para la defensa frente a los peligros ${ }^{14}$. Mas en el caso de emociones como la admiración carecemos de una base de juicio comparativa. Seguramente emociones como la admiración, la alegría y el amor pueden tener un uso instrumental, pero este no es central para el juicio acerca de la idoneidad del objeto. En razón de su papel para la constitución del valor, emociones como la admiración respaldan más bien concepciones emotivistas y disposicionales, las cuales subjetivizan más o menos radicalmente las emociones y dejan, por eso, solo poco espacio para el discurso sobre la idoneidad de las emociones.

Con esto nos encontramos nuevamente en el punto inicial. En relación con ciertas emociones como el miedo se vislumbra efectivamente una respuesta relativamente satisfactoria a la pregunta por su relación con los valores y su idoneidad, pero comparativamente esta respuesta es poco interesante. Por el contrario, allí donde las emociones parecen jugar un papel constitutivo importante para los valores, nos amenaza una recaída en concepciones que no le hacen justicia a importantes particularidades de nuestra relación valorativa del mundo ni a nuestra auto-relación.

\section{Una perspectiva evolutiva y social}

LA ASUNCIÓN DE UNA PERSPECTIVA EVOLUTIVA E HISTÓRICA nos podría señalar una salida a esta confusa situación. Podemos intentar esbozar un largo y complejo proceso con nuestro actual vocabulario valorativo, en calidad de fin provisional de tal proceso. Tal propuesta ha sido hecha por David Wiggins (1998, pp. 185-214) en su ensayo -inspirado en Hume-A Sensible Subjectivism? A diferencia de Wiggins, y por razones que expuse en el primer punto de la sección anterior, pienso que deberíamos permitir comenzar el proceso de constitución de determinadas adscripciones de valores con

\footnotetext{
${ }^{14}$ El temor puede ser exagerado no solamente cuando es la reacción a circunstancias que no representan peligro, sino también cuando la persona afectada se paraliza por el temor y debido a ello queda expuesta sin protección alguna al peligro.
} 
reacciones emocionales, las cuales no se cimentan sobre convicciones axiológicas o juicios de valor. Partiendo de esto, se puede suponer que objetos que regularmente dan lugar a una emoción como la admiración -0 un antecedente de ella- se agrupan gradualmente bajo la categoría común de lo admirable. De esta manera se podrían constituir casos paradigmáticos de lo admirable, los cuales suministran su contenido descriptivo a un predicado valorativo específico o compacto como admirable. En conexión con Ronald de Sousa, también se podría hablar de escenarios paradigmáticos, en los cuales la reacción emocional ante cierto objeto o tipo de objeto se conecta por primera vez o regularmente con la adscripción de una valoración fundada emocionalmente ${ }^{15}$. Pero no deberíamos interrumpir nuestro asunto en este punto; si debiéramos hacer eso, entonces una teoría valorativa disposicional sería tal vez la más apropiada. Como ya se admitió, ello puede ocurrir con predicados valorativos como repugnante y aburrido, pero en muchos otros casos el desarrollo puede ir más allá. Así, en un próximo paso y con ayuda de la determinación de similitudes familiares, el campo de los objetos admirables podría haber sido ampliado más allá de los objetos paradigmáticos. Y esto, por otra parte, podría haber conducido a un proceso de adaptación mutua entre las reacciones emocionales y la adscripción de los predicados valorativos que fueron presentados al comienzo. Esta posibilidad es el punto crucial. Por una parte, ella abre vías para la corrección de reacciones emocionales, en razón de percepciones refinadas y de juicios sobre los objetos a los cuales se refieren las emociones. Por otra parte, al mismo tiempo coloca el fundamento para la corrección y refinamiento de percepciones y juicios con respecto a una elevada sensibilidad hacia las correspondientes reacciones emocionales. Wiggins (1998, p. 198) menciona que nosotros podemos tomar en cuenta un objeto o clase de objetos, con el fin de argumentar en favor de la idoneidad de ciertas reacciones emocionales o, por el contrario, podemos escoger determinada reacción como punto de partida, y después aportar razones en favor de las características del objeto para las cuales la reacción parece estar hecha. Las experiencias emocionales y los juicios situacionales podrían haberse alimentado mutuamente tanto de esta manera, que al final ellos ya no podrían ser aclarados de forma independiente.

\footnotetext{
${ }^{15}$ De Sousa (1987, p. 181 ss.), establece puntos claves sobre dos aspectos: "first, a situation type providing the characteristic objects of the specific emotion-type [...], and second, a set of characteristic or 'normal' responses to the situation, where normality is first a biological matter and then very becomes a cultural one" (De Sousa, 1987, p.182).
} 
Por medio de la referencia a objetos paradigmáticos y con las correspondientes actitudes emocionales, el asunto desarrollado hasta ahora aporta importantes puntos de referencia para una mejor comprensión de la crítica relativa a la adscripción de valores y del discurso sobre la idoneidad de una emoción como la admiración. Sin ir al detalle, ella debe, sin embargo, ser complementada en -por lo menos- dos aspectos. Las fases críticas del proceso bosquejado son, por una parte, la determinación de los objetos paradigmáticos $\mathrm{y}$, por otra, la ampliación de su ámbito hacia nuevos objetos. ¿Cómo pueden estos procesos en realidad conducir al resultado? Para que ellos puedan afianzarse debemos, primero, asumir que hay suficiente homogeneidad en las reacciones emocionales de un grupo de seres humanos o en su disposición hacia tales reacciones. Para las emociones eso significa que un conjunto de ellas debe estar ajustado respectivamente a las características especiales del mundo. Nuestra adscripción de valores -que se ha fundado emocionalmente- carecería de toda clase de estabilidad y fiabilidad, si reaccionáramos siempre con diferentes emociones ante situaciones iguales o similares, y también si ante situaciones totalmente diferentes reaccionáramos con las mismas o similares emociones. Pero es dudoso que la estabilidad y fiabilidad necesarias puedan ser garantizadas solamente a través de patrones de actitud emocional determinados biológicamente. Por esta razón, son necesarias, en segundo lugar, formas de presión social y del entendimiento intersubjetivo, a los fines de poner en marcha y regular el establecimiento de paradigmas y su respectiva ampliación. Únicamente en virtud de la presión social y de los procesos de entendimiento intersubjetivo estamos en situación de fijar el significado de conceptos valorativos de tal manera que podemos intercambiar pareceres sobre ellos. La necesidad de hacer compresibles a los otros nuestras valoraciones fundadas emocionalmente, o inclusive compartirlas con otros, nos obliga a trascender nuestras variables $-\mathrm{y}$ con frecuencia altamente inconstantes- reacciones emocionales, sin que por ello deban llegar a ser superfluas, todo con vista a la constitución de valores. Hume tuvo en mente algo de este tipo cuando escribió en su Tratado sobre la Naturaleza Humana:

Además de que nosotros mismos cambiamos frecuentemente nuestra situación [...], cada día nos encontramos con personas que están en una situación diferente de la nuestra, y quienes no podrían nunca conversar con nosotros en términos razonables, si permaneciéramos constantemente en esa situación y en ese punto de vista que es propio de nosotros. De allí que la relación de los sentimientos, en la sociedad y en la conversación, hace 
que formemos algún estándar general inalterable, por el cual podemos aprobar o desaprobar caracteres y maneras. (Hume, 1989, p. 603)

Esta observación es adecuada para abrir todo un campo de investigaciones. Cómo puede ser concebido el proceso de constitución intersubjetiva que Hume delineó vagamente, es algo que en la filosofía se ha investigado muy poco. En este lugar no puedo satisfacer este desiderátum, pero quisiera, por lo menos, emprender una modificación del pensamiento de Hume. Ello atañe a la supuesta inmutabilidad de aquellos estándares sociales o intersubjetivos que tomamos como base para las valoraciones. Es decir, debemos contar con que al asunto de nuestro lenguaje valorativo pertenecen interpretaciones esencialmente discutibles, las cuales radican en diferentes -también emocionales- experiencias y que son expresión de diferentes concepciones de lo que es una vida buena para los seres humanos. Esto es aplicable al establecimiento de objetos paradigmáticos para las emociones particulares, pero tanto más válido para la ampliación del ámbito de estos objetos y su descripción detallada. Por eso es de esperar que algunas -si no la mayoría- de las disputas sobre valores no puedan ser concluidas sin que uno de los que toma parte en la discusión deba tener razón y el otro no ${ }^{16}$. Que se puedan aportar razones en favor de adscripciones -que están enraizadas en emociones- de predicados valorativos como admirable, no significa que estas razones deban ser concluyentes.

\section{Valores morales}

HASTA AHORA HE LLEVADO A CABO REFLEXIONES SOBRE la relación general de las emociones y los valores, y he discurrido sobre dos tipos de relaciones que hay entre emociones y valores: relación constitutiva y relación no-constitutiva. En el caso de las emociones que no son constitutivas de valores es posible formular escalas de valoración de su idoneidad o no idoneidad, sin que con ello se haga referencia a ellas mismas, pero esto no es viable en el caso de las emociones que son constitutivas de valores. Ahora quisiera referirme a los valores morales en tanto una clase especial de valores. ¿En qué medida pueden ser explicados sensatamente los valores morales con la ayuda de una concepción de los valores que da sustento a dichos valores morales sobre las ideas de las emociones idóneas?

\footnotetext{
${ }^{16}$ Aquí se debe ubicar una importante fuente de la inconmensurabilidad valorativa o inconmensurabilidad de valores.
} 
Para dar una respuesta a ello debemos saber, en primer lugar, qué es lo que debe contar como valor moral. Esto no es claro, ya que es discutible cómo la esfera de la moral puede ser delimitada de la mejor manera posible de otros ámbitos de nuestra vida. A los efectos de evitar disputas que son innecesarias para mis fines, quisiera tomar como base una comprensión más bien amplia y vaga de lo moral. Bajo esta condición, expresiones de las virtudes y de los vicios pueden ser tomadas como expresiones de los valores morales. De esta suerte, predicados como justo, generoso, benéfico, valiente, prudente e inteligente representan valores morales positivos, mientras que predicados como injusto, deshonesto, pusilánime, cruel y mezquino representan valores morales negativos. Las virtudes y los vicios a que con ellos se alude se descomponen sencillamente en dos grupos: algunos valen como requisitos para la vida buena del individuo, mientras que otros están conectados más claramente con nuestra convivencia con los otros. La prudencia es un claro ejemplo del primer grupo, mientras que la justicia y la beneficencia son ejemplos clásicos del segundo.

En un paso adicional, debemos considerar si es posible y tiene sentido catalogar como valores morales tan ilimitados las emociones particulares, en tanto que son reacciones adecuadas. En relación con esto, ya anticipé un resultado positivo cuando califiqué la injusticia y la crueldad como objetos naturales de la indignación. Pero no creo, en contraste con un grupo de autores, que haya emociones que tengan un sentido moral intrínseco, emociones que se puedan manifestar solo como respuestas ante el comportamiento moral correcto o incorrecto. También la indignación, el resentimiento y el sentimiento de culpa, que frecuentemente son reivindicados como las emociones verdaderamente constitutivas de la moral, pueden desencadenarse en contextos que no son de naturaleza moral, por lo menos en un sentido estricto $^{17}$. Además, también otros sentimientos como compasión, respeto, benevolencia y admiración juegan un papel significativo en nuestra vida moral. No obstante, ni lo uno ni lo otro son perjudiciales para la posibilidad de clasificar como valores morales las emociones particulares, en tanto formas de reacción apropiadas.

Pero con ello por sí solo no se ha ganado mucho. Seguramente podemos, por ejemplo, caracterizar la injusticia también como algo que merece

\footnotetext{
${ }^{17}$ Pueden encontrarse más detalles sobre este punto en Wildt, 1993, pp. 188-217.
} 
indignación o un sentimiento similar. Pero a la luz de las consideraciones sobre la diferencia entre temor y admiración, la pregunta decisiva es si los valores morales pueden ser concebidos como algo que de algún modo se constituye a través de determinadas emociones. Aun cuando este no es el caso, las emociones podrían asumir funciones importantes en el ámbito de la moral. En este sentido, emociones como indignación y sentimiento de culpa son, sin duda, útiles, si es que acaso no son incluso irrenunciables a los fines de la consolidación motivacional de las reglas morales. Y frecuentemente nuestras emociones nos ayudan a reconocer como moralmente cuestionable algo que sin ellas calificaríamos tal vez como carente de problemas ${ }^{18}$. Con todo, es fácil admitir estas funciones de las emociones dentro de la moral. Por el contrario, la tesis conforme con la cual las emociones establecen, por lo menos en parte, lo que significa ser moralmente bueno o malo, virtuoso o vicioso, es filosóficamente exigente, y debido a ello, naturalmente, también es algo muy discutible.

Ahora, a primera vista, hay más cosas que hablan en favor antes que en contra de la tesis de la constitución. Virtudes centradas en el yo, como lo son la perseverancia y la sensatez, son para nosotros ante todo de valor instrumental: las necesitamos para poder llevar una vida buena o en cierta medida satisfactoria. Ellas nos ayudan a manejar inteligentemente los obstáculos externos y los conflictos internos. Las elogiamos porque nos son útiles para la conducción de una vida que para nosotros es valiosa. Su valoración puede estar teñida de emocionalidad pero, así como en el caso del peligro y el temor, aquí también es válido lo siguiente: nuestra alabanza a la perseverancia y la sensatez no las convierten en virtudes. Lo positivo de ellas podemos explicarlo sin referencia a las emociones con las cuales, dado el caso, reaccionamos ante las mismas. Se trata inclusive del mismo patrón de medida que empleamos para la valoración de peligros y de las virtudes que se centran en el yo, o sea, el bienestar de los correspondientes individuos.

¿Pero no podemos decir algo semejante de virtudes sociales como justicia y beneficencia? También aquí es evidente que uno se debe remitir al bienestar de los particulares, de un grupo o de todos los seres humanos, en

\footnotetext{
${ }^{18}$ Aunque la mecánica contraria también puede tener lugar, por lo que las emociones pueden presentarnos como moralmente discutible algo que, visto más serenamente, es totalmente inocuo.
} 
tanto escala relevante. Para el bienestar de ciertos individuos o de muchos, las emociones podrían ser importantes, tal vez hasta constitutivas. Por ejemplo: en el caso de que no pueda haber una vida buena sin alegría. Pero la tesis de que las emociones son constitutivas para las virtudes y los vicios significa algo diferente: ella apunta a las emociones con las que reaccionamos frente a las virtudes y los vicios, y sostiene que esas emociones son constitutivas para los valores morales. Necesitamos una prueba en favor de esta tesis, lo que se dificulta porque las emociones en sí mismas pueden devenir en objeto de una valoración moral -en sentido amplio o restringido-, de tal forma que más bien la moral parece arrojar una luz sobre las emociones y no a la inversa.

Pero la tesis de la constitución, que ha sido sostenida en diferentes formas (verbigracia, por David Hume y Adam Smith), ¿no tiene ciertas ventajas? ¿Acaso los valores morales y ciertas emociones no están más entrelazados de lo que sugiere la referencia al papel motivacional de las emociones? Dos razones hablan en favor de una respuesta positiva.

Por una parte, los juicios morales y la propensión hacia ciertas emociones van de la mano en la formación de una conciencia moral. Para verdaderamente aprender a entender, por ejemplo, qué son la crueldad y la humillación, y por qué son malas, debemos tanto estar familiarizados con una cantidad de reacciones emocionales (por ejemplo, compasión y simpatía) como estar en capacidad de aportar argumentos para su justificación. Por lo menos en la fase de adquisición de un vocabulario moral, la comprensión de determinados valores morales pareciera ser una cuestión tanto de la sensibilidad emocional como de la percepción y la reflexión.

Por otra parte, una determinada comprensión general de la moral habla en favor de la exactitud - por lo menos parcial- de la tesis de la constitución. Cuando distinguimos las virtudes particulares -y los correspondientes vicios- en razón de su relación con el bienestar de algunos o de muchos, concebimos las virtudes fácilmente como medios para un fin que es diferente de ellas mismas y, luego, tomamos ese fin como escala para su valoración. Desde esta perspectiva, la moral se vuelve una especie de dispositivo para la promoción de fines extra-morales. Para mí, también es cierto que la moral es eso, mas ella no es únicamente eso. Hay ciertos vicios y virtudes que censuramos o elogiamos porque vemos en ellos disposiciones hacia formas de comportamientos, las cuales apreciamos o censuramos en sí mismas. Pero en el caso de una virtud centrada en el yo, como lo es la sensatez, nuestra 
valoración no se puede referir únicamente a la circunstancia de que ella contribuye con la persona para alcanzar un manejo satisfactorio de sus propios anhelos. Antes bien, podemos apreciar personas en razón de su sensatez sencillamente porque con ella se aproximan a un ideal de ser humano. Y cuando pensamos en vicios como crueldad y lealtad se manifiesta en ellas una actitud que podemos aborrecer o rechazar emocionalmente de cualquier otro modo. Es posible que este rechazo devenga en algo moral sólo cuando tenga lugar desde un determinado criterio o perspectiva (por ejemplo, desde la perspectiva del observador imparcial o la de todos los afectados por un acto). Pero aun así parece plausible decir que el demérito intrínseco a vicios como la crueldad y deslealtad se funda por medio de nuestra desaprobación emocional, entendida al menos en sentido amplio. Naturalmente, podemos condenar erróneo algo como cruel o desleal; nuestra sola condena no basta para hacer que algo cruel, desleal y moral sean indignos, ya que cruel y desleal son predicados compactos que siempre aluden a propiedades naturales sobre las cuales podemos equivocarnos. Pero cuando nuestra condena tiene como sustento informaciones y experiencias correctas, como se puede colegir de la historia de la valoración de algo en tanto cruel o desleal, entonces dicha condena es adecuada con respecto a su objeto y lo hace algo digno de ser condenado.

Esta perspectiva de los valores morales se hace más comprensible cuando la caracterizamos como algo cuasi-estético. Me parece que designar algo como bello, feo o excelso tiene sentido únicamente bajo la suposición de que hay seres que disponen de ciertas formas de sensibilidad emocional. Como se expuso en el ejemplo de lo admirable, ello no debe significar que algo merece ser cualificado como, por ejemplo, feo, únicamente cuando ello efectivamente es asimilado por algunos o muchos con un amplio desagrado. La caracterización por medio de predicados valorativos estéticos está abierta al intercambio de argumentos que puede apuntalarse en ese proceso socialevolutivo que, siguiendo a Wiggins, intenté esbozar. No obstante, en última instancia cualificar algo, por ejemplo, como feo permanece conectado con las reacciones emocionales. Y parece ser exactamente de esa misma forma en el caso de la valoración negativa de acciones en tanto crueles o desleales. Debido a su contenido descriptivo, podemos efectuar apreciaciones con estos predicados, sin que haya ningún tipo de participación emocional; y por ello también podemos aprender a juzgar acciones como crueles o desleales de modo emocional. Mas si no conectamos las acciones con una utilidad 
que es independiente de ellas, entonces su significado se remite, al fin y al cabo, a ciertas reacciones emocionales. Para decirlo enfáticamente: no hay, al final, ninguna razón de mayor peso para condenar la crueldad que nuestra animadversión frente a ella.

\section{Normas morales}

A PESAR DE ESTA EQUIPARACIÓN DE LOS VALORES MORALES con otros valores, especialmente con los estéticos (una equiparación que fue popular ante todo en la filosofía pre-kantiana del siglo XVIII), yo quisiera concluir con una observación que concierne a una particularidad de la moral, o sea, a una cierta rama de la moral.

Esta rama de la moral -la mayoría de las veces subsumida bajo el concepto de justicia- se refiere a cosas que debemos a los demás. La moral exige algo de nosotros, y no en pocas ocasiones creemos que las exigencias morales, en caso de conflicto, deberían superar todos los demás intereses. Lo que debemos a los demás son cosas que nosotros debemos hacer, y esto no es cuestión de los valores morales, sino de las normas morales. Pero las normas poseen una lógica diferente de la de los valores; para ir de los valores a las normas siempre se debe ejecutar un paso que no se da sencillamente por sí mismo. Presumo que el paso se corresponde en cierta manera con el que debemos efectuar sobre la base de experiencias emocionales, con el fin de formar la volición y las intenciones. A la luz de la volición y las intenciones, algo se nos manifiesta como bueno o malo, pero que nuestro actuar luego se realice, depende de decisiones que podemos tomar recurriendo a experiencias emocionales, las cuales, sin embargo, normalmente no fuerzan las decisiones (Steinfath, 2001, cap. 5).

Ya señalé que frecuentemente justificamos normas en consideración de los valores. Por ejemplo, la norma que prohíbe causar sufrimiento a otros seres humanos solo por el propio placer podemos justificarla con referencia a la circunstancia de que una violación de esta norma sería algo cruel o abominable. Los mandatos y las prohibiciones morales deben sustentarse sobre algo, y los valores morales son el mejor candidato para ello. Pero estos pueden colidir con otros valores, sean morales o no. Luego, estamos obligados a ponderar los valores unos con otros. No conozco ninguna pauta sencilla de acuerdo con la cual se pueda proceder, y tengo profundas dudas 
de que exista tal pauta. Ello implica que considero erróneo el criterio según el cual las consideraciones morales siempre deben prevalecer frente a todas las demás consideraciones.

Aquí no puedo profundizar en ese tema. Pero es importante tomar en cuenta que la transición de los valores morales hacia las normas morales $-\mathrm{y}$ de cualquier valor a las normas en general- presenta una complicación. Por eso, una teoría de los valores únicamente puede ser un primer paso para la comprensión de la moral. Aunque una teoría que se derive de las reflexiones precedentes sobre una posible relación constitutiva de los sentimientos y los valores también tiene importantes efectos sobre la comprensión de la moral. Allí donde la adscripción de determinados predicados valorativos se debe a un proceso socio-evolutivo, proceso en el cual las reacciones emocionales, las percepciones y los juicios se fecundan mutuamente, allí la crítica y la fundamentación de los valores únicamente puede ser por principio interna. La crítica y fundamentación adherirá al proceso que es obligatorio para cierta práctica social, a fin de fijar el significado de los predicados valorativos individuales y, también, adherirá a la posibilidad de deber apelar a ciertas experiencias emocionales. El relativismo que allí yace, y que se agudiza con la posibilidad de hacer interpretaciones divergentes de casos paradigmáticos en las atribuciones de valores individuales, no se opone a un universalismo parcial y empírico. Es posible que haya modelos de reacciones emocionales -sobre las cuales se construyen ciertas adjudicaciones de valor- y que estos modelos sean comunes a todos los seres humanos. Pero se debe partir de que hay valores morales y valores no morales que se encuentran condicionados cultural e inclusive individualmente y que, por consiguiente, varían. 


\section{Referencias}

Anderson, E. (1995). Value in Ethics and Economics. Cambridge: Harvard University Press.

Ayer, A.J. (1936). Language, Truth and Logic. London: Gollancz.

Aristóteles. (1976). Ars Rhetorica. Berlin: De Gruyter.

Bedford, E. (1957). Emotions. Proccedings of the Aristotelian Society, 57, pp. 281-304.

D’Arms, J. \& Jacobson, D. (2000). Sentiment and Value. Ethics, 110(4), pp. 722748.

De Sousa, R. (1987). The Rationality of Emotion. Cambridge: The Massachusetts Institute of Technology.

Hume, D. (1988 [1738]). A Treatise of Human Nature. Oxford: Oxford University Press.

Johnston, M. (2001). The Authority of Affect. Philosophy and Phenomenological Research, 63(1), pp. 181-214.

Lewis, D. (1989). Dispositional Theories of Value. Proccedings of the Aristotelian Society, 63, pp. 113-137.

Lyons, W. (1980). Emotion. Cambridge: Cambridge University Press.

Mackie, J.L. (1977). Ethics. Inventing Right and Wrong. Harmondsworth: Penguin Books.

McDowell, J. (1998). Mind, Value and Reality. Cambridge: Harvard University Press.

Millgram, E. (1995). Inhaltsreiche Begriffe und Unterscheidung zwischen Werten und Tatsachen. C. Feige \& G. Meggle, G. (Hrsgs.), Zum Moralischen Denken (Tom. 1, pp. 354-388). Frankfurt am Main: Suhrkamp.

Mulligan, K. (1998). From Appropiate Emotions to Values. The Monist, 81(1), pp. 161-188.

Nussbaum, M.C. (1996). Upheavals of Thought. The Intelligence of Emotions. Cambridge: Cambridge University Press. 
OAKLEY, J. (1992). Morality and the Emotions. London: Routledge.

Peacocke, C. (2001). Does Perception Have a Nonconceptual Content? The Journal of Philosophy, 98(5), pp. 239-264.

Pitcher, G. (1965). Emotion. Mind, 74, pp. 326-346.

Solomon, R. (1993). The Passions. Indianapolis: Hackett Publishing Company.

Scheler, M. (1980 [1916]). Der Formalismus in der Ethik und die materiale Wertethik. Neuer Versuch der Grundlegung eines ethischen Personalismus. Bern/ München: Francke.

Smith, A. (1984 [1759]). The Theory of Moral Sentiments. Indianapolis: Liberty Fund.

Steinfath, H. (2001a). Orientierung am Guten. Praktisches Überlegen und die Konstitution von Personen. Frankfurt am Main: Suhrkamp.

Steinfath, H. (2001b). Gefühle und Werte. Zeitschrift für philosophische Forschung, 55(2), pp. 196-220.

Stocker, M. (1996). Valuing Emotions. Cambridge: Cambridge University Press.

Wiggins, D. (1998). A Sensible Subjectivism? Needs, Values, Truth (pp. 185-214). Oxford: Oxford University Press.

Wildt, A. (1993). Die Moralspezifität von Affekten und der Moralbegriff. H. Fink-Eitel \& G. Lohmann (Hrsgs.), Zur Philosophie der Gefühle (pp. 188-217). Frankfurt am Main: Suhrkamp. 